\title{
Alternative Methods for Studying Consumer Payment Choice
}

\section{Oz Shy}

\section{Working Paper 2020-8a June 2020 (revised November 2022)}

\begin{abstract}
The study of consumer payment choice at the point of sale involves classifications of payment methods such as cash, credit cards, debit cards, prepaid cards, checks, and electronic payments withdrawn from consumers' bank account. I compare alternative methods for studying consumer payment choice using some machine learning techniques applied to the 2021 survey and diary data on consumer payment choice. The results are then compared to the more traditional logistic regression methods. Machine learning techniques have advantages in generating predictions of payment choice, in visualization of the results, and in application to high-dimensional data. The logistic regression approach has an advantage in interpreting the probability that a buyer uses a specific payment instrument.
\end{abstract}

JEL classification: C19, E42

Key words: studying consumer payment choice, statistical learning, machine learning https://doi.org/10.29338/wp2020-8a

The author thanks participants at the Bank of Canada Retail Payments Workshop held in 0ttawa 0ctober 24-26, 2018, for valuable comments on an earlier draft. The views expressed here are those of the author and not necessarily those of the Federal Reserve Bank of Atlanta or the Federal Reserve System. Any remaining errors are the author's responsibility.

Please address questions regarding content to 0z Shy, Research Department, Federal Reserve Bank of Atlanta, 1000 Peachtree St. NE, Atlanta, GA 30309, oz.shy@atl.frb.org.

Federal Reserve Bank of Atlanta working papers, including revised versions, are available on the Atlanta Fed's website at www.frbatlanta.org. Click "Publications" and then "Working Papers." To receive e-mail notifications about new papers, use frbatlanta.org/forms/subscribe. 


\section{Introduction}

Data on "how consumers pay" are collected by consumer surveys in which consumers list all the payment instruments they have and whether and how they use them at the point of sale (POS). There are two types of consumer survey: Surveys that rely on memory recall (how many credit card payments have you made during the last year or month?) and diary surveys in which consumers record, either in real time or by the end of each day, all payment related activities including dollar amount, spending type, merchant type, and payment method. For budgetary reasons, consumer diaries (used in this paper) are limited to few thousands respondents thereby generating approximately between fifteen to thirty thousand payment observations. Whereas these numbers are considered to be "small data" relative to payment data collected by Visa, MasterCard, Amazon, and other retailers that collect "big data," consumer diary surveys are more suitable for analyzing consumer payment choice because they provide information on a wide variety of spending types using all payment methods rather than just credit and debit card payments.

The study of consumer payment choice at the point of sale involves classifications of payment methods such as cash, credit cards, debit cards, prepaid cards, checks, and electronic payments withdrawn from consumers' bank account. Because these studies focus on classification of payment methods, researchers have traditionally relied on discretechoice analyses using binomial, multinomial, logit, and probit regression methods. These studies measure mainly the effects of dollar transaction value (amount), demographic variables, and spending/merchant type on the probability that a consumer chooses to pay with a particular payment method such as credit card instead of cash.

Whereas standard regression models will remain a powerful tool for studying payment choice, the goal of this article is to explore alternative and somewhat newer methods involving machine (statistical) learning techniques. Machine learning methods introduce new insights to our attempts to predict how a specific consumer pays for a specific transaction at a particular POS, and these techniques become essential when using "big data."

This article compares a few machine learning classification techniques and the more 
transitional multinomial regression technique. Such comparisons have been made many times before. One of the earlier papers to analyze and compare the techniques dates back to a seminal paper by Breiman (2001) who describes and compares the predictive performance of machine learning algorithms and logistic regression on multiple datasets. That paper also provides extensive discussions on the differences between the "two cultures:" the model estimation approach and the algorithmic approach to data analysis. Unlike the above mentioned comparisons, the novelty of this article is the comparison of these techniques specifically in the context of consumer payment choice with the use of payment choice diary data.

Kleinberg et al. (2015) distinguishes between "pure prediction problems" where the researcher's only goal is to accurately predict $Y$ given $X$, and the more typical case in economics where the researcher is interested in learning how $X$ affects $Y$ and therefore estimating the parameters of a model. As noted earlier, most research on payment choice focuses on estimating parameters of a model with the goal of, for example, learning how the probability of paying with a credit card changes between consumers with $\$ 60,000$ household income and consumers with $\$ 70,000$ household income. In contrast, the present article puts less emphasis on causal effects and instead focuses on prediction accuracy of the choice of a payment method given the payment amount and the payer's demographic features.

The recent adoption and use of machine learning techniques in economics, marketing, and sales have been discussed in Varian (2014), Bajari et al. (2015), Mullainathan and Spiess (2017), and references therein. The statistical properties of these techniques are discussed over the Internet and in several textbooks, such as James et al. (2013). Finally, Hillel et al. (2021) conducts a systematic review of machine learning methodologies for modelling passenger mode choice and identify several methodological limitations (some of them may apply to the analysis that I conduct in this paper).

The list below characterizes the payment methods collected in the diary survey. Other variables in the data are described in section 2 . These characterizations have been pro- 
vided to all the respondents who participated in the survey and diary of consumer payment choice.

\section{Electronic payment methods:}

- Bank account number payment (BANP): You [the respondent] give your bank routing number and account number to a third party to authorize a deduction from your bank account.

- Online banking bill pay (OBBP): You initiate a payment at your bank's online banking website.

- Account-to-account money transfers.

- Income deduction: Your employer makes the payment for you on your behalf and deducts the payment amount from your salary.

\section{Card payment methods:}

- Debit card: Your payments are deducted from your bank account. Also, you can use a debit card to withdraw or deposit cash at ATMs.

- Credit card: You pay back the credit card company later. Credit cards charge interest (unless the balance is paid by the initial due date).

- Prepaid/gift/EBT card: You store or load money on a prepaid card. Sometimes called "prepaid debit," "gift cards," "payroll cards," or "stored value cards."

\section{Paper payment methods:}

- Cash: Coins and paper bills.

- Check: You write a paper check to a person or business.

- Money order: You purchase a money order from a bank, post office, check-cashing store, or retail store. At the time of purchase, you specify the amount and the person or business to be paid.

The analysis in this article will be confined to the eight payment methods with the highest share of use. 
This article is organized as follows. Section 2 describes the data and defines the variables of interest. Section 3 presents classification trees and shows how they can be used to visualize and predict consumer payment choice. The predictions are then compared to predictions generated by multinomial regressions. Section 4 extends the classification tree analysis to random forest. Section 5 introduces the $k$-nearest neighbors algorithm. Section 6 illustrates the support vector machine algorithm. Section 7 concludes. The data and R-code used in this analysis are available for downloading from https://github.com/ozshy/diary ml.

\section{Data, variable selection, sample statistics, and coding}

The data are taken from the 2021 Survey and Diary of Consumer Payment Choice (SDCPC). ${ }^{1}$ The SDCPC uses a representative sample of US (18 and older) consumers. The SDCPC records transactions during three consecutive days. Transactions include purchases, bill payments, ATM withdrawals, and deposits. Respondents' three-day diaries were evenly distributed throughout the month of October 2021 in a way that resembles a three-period overlapping generations model. The SDCPC collects a large number of variables describing all sorts of demographics and transactions. For the purpose of this article, I focus on only a small subset of variables.

Table 1 displays demographic information of 3,137 respondents. Weighted data are not used in the analysis and are brought here in order to demonstrate the similarities and differences between the sample variables and the actual US adult population.

Table 2 shows that out the 14,135 total payments made by 3,137 respondents, twothirds (66.1 percent) were paid "in-person" and one-third (33.9 percent) were paid "remotely." Cash payments constitute 27.7 percent of in-person payments, debit card payments (31.7 percent), and credit card payments (33.8 percent). Remote payments were

\footnotetext{
${ }^{1}$ The Federal Reserve Banks of Atlanta, Boston, and San Francisco conduct the diary survey. The results are summarized in Cubides and O'Brien (2022) and Foster, Greene, and Stavins (Forthcoming). Similar surveys are conducted by the Bank of Canada; see Henry, Huynh, and Welte (2018). The data and assisting documents (codebooks) are publicly available for downloading from the Federal Reserve Bank of Atlanta website: https://www.frbatlanta.org/banking-and-payments/consumer-payments.aspx.
} 
made mostly with debit cards (24.5 percent), followed credit cards (21.9 percent), BANP (20.9 percent), OBBP (19.5 percent), and checks (7 percent).

A note about terminology. In regression analysis, the explanatory variables are also called independent variables, explanatory variables, regressors, or right-hand side variables. In machine learning, the same variables are often called features, attributes, predictors, or input variables. The reader should bear in mind that the analysis in this paper uses all these terms to mean the same thing.

The remainder of this section describes the eight numerical and categorical variables (features) that are used in the analysis. ${ }^{2}$

- Amount: Numeric payment amount ranging from $\$ 0.16$ to $\$ 25,000$, with median $\$ 33.00$ and mean $\$ 145.92$.

- In-person: a categorical variables indicating whether the respondent paid in-person (9,347 payments or 66.1 percent) or remotely (4,788 payments or 33.9 percent). Remote payments are generally made online, deduction from a bank account, or via the mail.

- Age: Integer-valued ranging from 18 to 111, with median 51 and mean 51.35.

- Gender: 1,831 (58.4 percent) female respondents and 1,306 (41.6 percent) male respondents.

- Marital status: 2,124 (67.7 percent) married, 1,013 (32.3 percent) not married.

- Education: 101 (3.2 percent) have less than high school, 1,327 (42.3 percent) have high school, 1,052 (33.5 percent) have an associate or a college degree, and 657 (20.9 percent) have MA or higher.

- HH income: Household income with median $\$ 75,000$ and average $\$ 106,250$.

- HH size: Number of people in the household ranging from 2 to 12 with median 3 and mean 3.5.

The analysis in this article does not apply demographic sampling weights because

\footnotetext{
${ }^{2}$ I consolidated the number of levels within some categorical variables. For examples, the number "Education" levels was reduced from 16 to 4 which makes it easier to interpret the results. The number of "Marital" levels was reduced by combining divorced, separated, and widowed into "not married."
} 
the whole purpose of this demonstration is to analyze the effects of demographics on payment choice (among other variables). In addition, because the goal is to compare very different statistical methods using the same data, I made sure that all these comparisons are conducted with the same training and testing subsamples which are sampled using the same pseudo-random number generating function (the set.seed $(N)$ command in R).

\section{Classification trees}

In the context of machine learning, a classification tree displays an optimized algorithm in the form of an upside-down tree. The tree illustrates how the machine (software) splits and classifies the data with the objective of minimizing a function of the number of classification errors among the predicted payment methods relative to the actually used payment methods.

The dataset is split into two separate subsamples: a training subsample and a testing subasmple. The classification tree algorithm is designed and tuned on the training subsample using cross validation. The cross validation procedure partitions the training subsample into $k$ folds, where the algorithm is constructed using $k-1$ folds of data and tested on the retained $k^{\prime}$ s fold on which the classification errors are measured. The process repeats itself $k$ times, each with a different retained $k^{\prime}$ s fold. The $k$ error measurements are then averaged. The advantage of this method is that all observations in the training subsample are used for both training and validation. The algorithm is tuned to produce the smallest average error via the complexity parameter $(c p)$ which controls the number of splits and hence number of branches of the classification tree. Parameters used for tuning (such as the $c p$ ) are often referred to as hyper-parameters because they are used for regularizing the fitting algorithm.

The resulting tree algorithm is then evaluated by measuring the classification errors generated by predictions made on the testing subsample which was held out separately from the training subsample and the cross validation procedure. That is, testing data constitute a subsample from the same population but with observations that were not 
included in the sample used for fitting the model. Therefore, the predictive power of a model is assessed on a subsample of the original dataset which is different from the training subsample that is used for the construction of a classification tree; a practice sometimes referred to as the "firewall principle."

To be able to compare the predictive power of the tree algorithm to that of logistic regressions, I duplicate the procedure by estimating the regression coefficients using the same training subsample that is used for designing the tree, and then measure the classification errors of the logistic regression model on the same testing subsample as the tree algorithm. Therefore, the sample with 14,135 payment observations described in section 2 is randomly split so that 11,308 (80 percent) of the payment observations are classified as training data, and the remaining 2,827 (20 percent) as testing data. The same split is retained throughout the analysis in order to be able to compare the efficiency (error minimization) of the various algorithms that are analyzed. ${ }^{3}$

In addition, the classification tree algorithm and the logistic regression are compared using exactly the same payment method classifications and the same explanatory variables that were described in section 2. The relationship between the choice of a payment method and the explanatory variables (features) is defined by the model

$$
\begin{aligned}
& \text { Method }_{t}= \alpha_{m}+\beta_{T} \text { Amount }_{t}+\beta_{P} \text { in-person }_{t}+\beta_{A} \text { Age }_{t}+\beta_{G} \text { Gender }_{t} \\
&+\beta_{M} \text { Marital }_{t}+\beta_{E} \text { Education }_{t}+\beta_{I} \text { HH_income } \\
& t
\end{aligned}
$$

where the dependent variable "Method" (cash, check, debit card, credit card, prepaid card, BANP, OBBP, and account-to-account) is the payment method used for transaction (payment) $t$ in the sample. The payment amount and the demographic variables (features) reflect those of the respondent who paid for transaction $t$.

\footnotetext{
${ }^{3}$ The data are split according to payment observations. However, because each respondent may make several payments, it is possible that some respondents end up in both the training and testing datasets. This may result in some data leakage from the training dataset to the testing dataset, see Hillel et al. (2021). Therefore, researchers who analyze samples with more than one payment observation per respondent may consider splitting the sample according to respondents rather than by payment observations.
} 


\subsection{Multiple payment methods classification tree}

Figure 1 displays an upside-down tree that classifies the use of eight payment methods according to the model defined in (1). For the purpose of this illustration, the tree drawn in Figure 1 has been excessively pruned compared with the optimal tree size. That is, the displayed tree has been pruned to have only 7 splits whereas the tree that minimizes the average cross-validated error (plus one standard deviation) should have about 23 splits, which are hard to fit into a single page. ${ }^{4}$

Figure 1 shows that that the "In-person" feature is the strongest predictor for separating cash from noncash payments. This is because cash must paid in person whereas card payments can be made both remotely and in-person and payments via BANP and OBBP are remote only. Note that the tree algorithm selects the "In-person" feature as the top split because this feature reduces classification errors more than a top split according dollar amount, age, or income (that are pushed to lower branches of the tree).

The second layer of branches in Figure 1 consists of splits according the payment dollar amount. For in-person payments, Figure 1 shows that a payment amount lower than $\$ 10$ constitutes the next-best predictor for consumers' choice of paying with cash, which explains 17-percent of all respondents' payment observations. The exact splitting amount ( $\$ 10$ in this sample) is determined by some function of the classification errors in the sense that any other split would generate lower prediction accuracy as measured by a function of the number of prediction errors. ${ }^{5}$ For remote payments, payment amounts between $\$ 10$ and $\$ 90$ are predicted to be paid with debit cards ( 7 percent) and credit cards (11 percent).

\footnotetext{
${ }^{4}$ The classification tree displayed in Figure 1 was constructed with the rpart R-package. Initially, the complexity parameter was set to $c p=0.001$, thereby generating 39 splits. For a given $c p$ value, the program attempts only the spits that increase the overall fit by a factor of $c p$ or higher. Then, in order to increase prediction accuracy, the complexity parameter was increased to $c p=0.001470292$ thereby reducing the number of splits to 23 . Note that this $c p$ value for this tree model only locally minimizes the xerror in the sense that lower $c p$ values further reduce the xerror. For the sake of illustration, the number of splits in Figure 1 was reduced to 7 using $c p=0.005387714$.

${ }^{5}$ The reader may wonder why the tree algorithm selects the $\$ 10$ payment amount as the optimal split (and not $\$ 9$ or $\$ 11$ ). This may have to do with the dollar denomination structure which causes consumers to carry $\$ 5, \$ 10$, and $\$ 20$ bills in their pocket. Discontinuities of this type have been analyzed in Shy (2020) using US diary data and in Chen, Huynh, and Shy (2019) using Canadian data.
} 
Higher than $\$ 90$ are predicted to be paid via OBBP (15 percent).

The third layer of branches in Figure 1 consists of splits according to education. The level of education (which is correlated with household income) predicts whether the payment will be made with a credit card or a debit card. Note that high household income is needed in order to obtain and use credit cards. This explains why associate degree or college degree and higher predict credit card payments because these respondents also earn higher household income. Thus, in case of credit cards, the choice of paying with a credit card may be implied by a supply-side effect whether the credit card issuer was willing to approve the credit card application by this respondent.

The analysis of classification trees has so far focused on the visualization aspect. The analysis below demonstrates the predictive aspect of the classification tree algorithm. I first construct a longer-than-optimal classification tree using cross validations restricted to the training subsample by setting a low $c p$ value. Then, the tree is pruned by increasing the value of the $c p$ parameter which reduces the number of splits to the level that minimizes the average cross-validation error.

Note that the reason why trees are pruned is to avoid the consequences of "overfitting." That is, longer trees reduce prediction error rates on the training data, but increase the prediction error rates when used over the testing data, and therefore result in poor predictive power over new data. To see that, suppose we construct a very long tree in which each observation results in its own terminal node. This tree achieves the maximum possible fit over the training data, but will surely fail to provide proper predictions using the testing data. For this reason, the long tree should be pruned using the desired $c p$ parameter. The pruned tree is then used to make predictions based on the testing data or any other new data from the same population.

Table 4 exhibits the confusion matrix (also called error matrix). The values on the main diagonal are the numbers of correct predictions based on the testing data. That is, the optimally-tuned tree correctly predicted 258 cash payments, 0 check payments, 456 debit card payments, 484 credit card payments, 0 prepaid card payments, 32 BANP payments, 
67 OBBP payments, and 0 account-to-account payments. The failure to predict any check, prepaid, and account-to-account payments stems from the fact that the tree splitting algorithm is heavily influenced by the relative number of observed payments made with each payment method. Therefore, splitting the features according payment methods that are not used very often in the sample does not yield sufficient reduction in the error rates, and consequently are not predicted by the algorithm.

The values that are not on the main diagonal in Table 4 reflect incorrect predictions. For example, 72 predictions that consumers pay with credit cards were incorrect because these consumers actually paid cash. Similarly, 142 predictions that consumers pay cash were incorrect because these consumers actually paid with credit cards.

The bottom row in Table 4 computes the tree algorithm's correct prediction rate for each payment method. It shows that out 536 actual cash payments in the testing data, the tree was able to predict only 48 percent accurately. Out of 837 credit card payments in the testing data, the tree predicted 58 percent accurately. Debit card payments were correctly predicted 54 percent, and as mentioned above, there were zero correct predictions of check, prepaid card, and account-to-account payments due to their relative low use.

\subsection{A comparison with multinomial regressions}

Multinomial regressions are commonly used in payments research. Therefore, I now compare the prediction accuracy of a multinomial regression to the prediction accuracy of the tree algorithm, using the exact same model defined in equation (1). The regression is run on exactly the same training subsample of 11,308 payments as the classification tree. The predictions are then performed on the same testing subsample with the same 2,827 payments as the classification tree. This is needed in order to be able to compare the number of prediction errors between the two algorithms.

Table 3 displays the estimated coefficients (not the marginal effects) of the multinomial regression. To maintain consistency throughout this article, $\mathrm{HH}$ income is measured in dollar units which explains why the coefficients are so tiny. Because the focus of this arti- 
cle is on prediction accuracy, I skip the lengthy discussions related to the interpretations of the estimated coefficients. For example, Table 3 shows that having a graduate degree $(\mathrm{MA}+)$ increases the log odds of paying with a credit card relative to cash by 2.98 . Similarly, paying in person decreases the log odds of paying with a credit card relative to cash by 2.55 because, as will be shown by other algorithms, paying in person is the strongest predictor for paying cash.

Moving on the predictions, Table 5 presents the confusion matrix and the prediction accuracy rates for the multinomial model. ${ }^{6}$ Comparing Table 5 with Table 4 shows that the classification tree algorithm has higher prediction accuracy rates for cash payments (48 versus 30 percent), debit card payments ( 54 versus 49 percent), and OBBP (36 versus 23 percent). However, the classification tree yields slightly lower accurate prediction rates of check payments ( 0 versus 6 percent), credit card payments (58 versus 62 percent), and BANP (16 versus 17 percent).

Therefore, we cannot conclude which algorithm predicts better at least for this sample. Classification trees have the advantage that they generate intuitive diagrams of payment method classifications, such as the one shown in Figure 1. However, regression coefficients (especially marginal effects) provide very useful intuition of the model parameters.

\section{Random forest}

Random forest algorithms do not generate classification trees like the one plotted in Figure 1. Instead, they build a large number of classification trees where each tree splits the training data according to a randomly-selected subgroup of features. The number of features in each subgroup is controlled by the researcher, and is generally chosen to be near the square root of the total number of features. More specifically, the specification of the model in equation (1) analyzes the data with the use of eight features. Therefore, each tree in the forest will split the data according to three randomly-selected features. This procedure decorrelates the sampled trees in the sense that strong features (such as

\footnotetext{
${ }^{6}$ Results were obtained using the multinom function in the nnet R-package.
} 
"In-person" and"Amount" in our data), will not be chosen as top splits in every tree.

Although random forest algorithms do not yield trees that can be drawn, they provide an important procedure for variable selection (in addition to improved prediction accuracy). Random forest algorithms rank the relative importance of the features for prediction purposes. Figure 2 exhibits a variable importance plot (VIP) generated by R's randomforest package. It shows that removing the predictor "In-person" from the model would increase the average number of misclassifications by 266 . Removing the predictor "Amount" from the model would increase the average number of misclassifications by 219. As the figure shows, "Marital" status is the least important variable because without it the average number of misclassifications would increase by 95 only. ${ }^{7}$

Most importantly, random forests are extremely useful for making predictions. The model defined in (1) is now applied to the random forest algorithm. The algorithm uses exactly the same training subsample containing 80-percent of the observations that were used to fit the classification tree and the multinomial regression analyzed in section 3. The algorithm's prediction accuracy is then tested on the same remaining 20-percent of the data. The resulting confusion matrix for the random forest predictions is given in Table 6.

Comparing Table 6 with Tables 4 and 5 reveals significant improvements in prediction accuracy for all eight payment methods. In particular, it predicted accurately 54 percent of cash payments, 68 percent of debit card payments, and 70 percent of credit card payments. It also shows a significant improvement in the prediction of check payments (33 percent versus 6 percent that were predicted by the multinomial regression and 0 percent by the tree algorithm). Note that check payments are hard to predict because are used less frequently relative to cash, debit, and credit cards; however, despite the low use, the random forest algorithm generated one-third accuracy rate.

Perhaps the most striking result is that, unlike the tree algorithm and multinomial,

\footnotetext{
${ }^{7}$ Figure 2 provides an easy visual framework for variable selection. Other commonly used variable selection methods include Lasso and Ridge regressions and principal component analysis that can be applied via several R-packages.
} 
random forest is the only prediction algorithm that generated some accurate predictions for prepaid card payments and account-to-account transfers. More precisely, random forest predicted correctly 6 out of 58 prepaid card payments and 7 out of 34 accountto-account transfers whereas the classification tree and multinomial regression predicted none. Although these prediction rates may seem to be low, the reader should bear in mind that they were made on the testing subsample which contains totally different payment observations than the training subsample that was used to fit these algorithms.

The reason for the significant improvement in prediction accuracy is that the random forest algorithm averages the predictions made by hundreds of trees, where each tree is constructed based on only three out of eight features. Therefore, dominant features, such as "In-person" and "Amount", are not always used, thereby letting a large number of trees be split based by less-dominant features. This exercise shows, perhaps paradoxically, that introducing more randomness into the prediction algorithm tends to improve prediction accuracy.

\section{5. $\quad k$-nearest neighbors $(k \mathbf{N N})$}

The $k$-nearest neighbors is an algorithm that inputs available observations and classifies (predicts) new choices based on a similarity measure in the space of features. Similarity is determined by the distance between the features of the observations. The $k \mathrm{NN}$ algorithm determines a neighborhood in the space of features for each observation in the training data. Each neighborhood contains $k$ observations, where $k$ serves as a tuning (hyper) parameter to be determined by the researcher.

Predictions of which payment method will be used are based on measuring the distance in the space of features to the $k$ nearest neighbors, and determining the payment method associated with the majority of these $k$ nearest observations. For example, if $k=3$, the algorithm measures the distance between the features of the new observation to its $k=3$ closest neighbors. If two of the its neighbors (say from the training subsample) are credit card payments and one is a cash payment, the new observation will be 
classified as a credit card payment by a simple majority rule. ${ }^{8}$

One drawback of the $k \mathrm{NN}$ algorithm is that it can analyze only numerical features because neighborhoods are defined according to distances among observations within each neighborhood (such as the Euclidean distance). This is unfortunate because most of the data collected in payments research contain categorical features such as merchant type and payers' demographic attributes. A partial solution to this drawback would be to turn categorical features into numerical. Therefore, for the kNN analysis, "In-person," "Married," and "Male" are assigned the value of 1 (versus 0 ), and the four levels of "Education" are assigned the values of $0, \frac{1}{3}, \frac{2}{3}$ and 1 .

Another potential problem that may arise from the fact that neighborhoods rely on distance measurements. Therefore, changing the units in which features are measured may alter the neighborhoods which are determined by this algorithm. For this reason, all the features are normalized to take values between 0 and 1 before running the $k \mathrm{NN}$ algorithm. That is, each numerical feature, for example a particular household income $\widehat{x}$, is normalized according to $[\widehat{x}-\min (x)] /[\max (x)-\min (x)]$, for each household income $\widehat{x}$ in the sample.

\subsection{Illustration of the $k \mathrm{NN}$ algorithm using two features}

The grouping of training payment observations into neighborhoods in the space of features cannot be plotted in two dimensions when there are more than two features. This is unfortunate because the algorithm is suitable for making predictions using all the eight features that are listed in the model defined in (1). Therefore, in order to illustrate these neighborhoods, I focus only on two numerical features: payment dollar amount (Amount) and household income (HH income). These two features are normalized to take values between 0 and 1 to avoid the influence of units of measurements.

In order to avoid dealing with the categorical "In-person" feature which can take only

\footnotetext{
${ }^{8}$ To some degree, $k \mathrm{NN}$ resembles clustering analysis ( $k$-means) which partitions the space of features into clusters according to distance (such as the Euclidean distance). Clustering analysis is useful for unsupervised data which contain only features and no response variables. The data analyzed in this article is supervised because the choice of payment methods (the response variable) is observed.
} 
two values, I also restrict this illustration to 9,347 in-person payments. This subsample of in-person payments is then divided into 7,477 (80 percent) training observations and 1,870 (20 percent) testing observations.

The top panel in Figure 3 depicts the actual 1,870 payment observations in the test data. To avoid cluttering, the axes are stretched by taking the logarithm of the normalized household income and payment amounts. The top panel shows that consumers with lower household income use cash more intensively than consumers with higher income. Cash is also used more often for low dollar amounts. Checks are used for large dollar amounts. Credit cards are used more intensively by consumers with higher household income.

The bottom panel in Figure 3 depicts 1,870 payment methods that were predicted from the two features (Amount and $\mathrm{HH}$ income) in the testing data, based on the neighborhoods determined by the $k \mathrm{NN}$ algorithm using the training subsample. In this exercise, the optimal (error-reducing) neighborhood size was found to be $k=19$ observations. The bottom panel shows that the $k \mathrm{NN}$ algorithm predicts less cash payments and more credit card payments by consumers with very high household income relative to the actually observed cash and credit card payments in the top panel.

\section{$5.2 k N N^{\prime} s$ predictions of payment methods based on all eight features}

I now apply the $k \mathrm{NN}$ algorithm to the same training subsample containing 11,308 (80percent) payment observations and test its prediction accuracy on the 2,827 (20-percent) payment observations in the testing subsample. ${ }^{9}$

Table 7 displays the confusion matrix for the payment method predictions made by the $k \mathrm{NN}$ algorithm applied to the same testing data used for the other three algorithms

\footnotetext{
${ }^{9}$ This analysis applies the knn function in the class R-package. This function integrates the training data classification (first step) and the predictions on the testing data (second step) into a single function. The parameter $k$ (number of observations in each neighborhood) is tuned by running a loop over this function for all values of $k=1, \ldots, 40$, yielding $k^{\mathrm{min}}=30$ as the value that minimizes the classification error rate. Note that each time $\mathrm{R}$ runs this code, it may generate a slightly different value of $k^{\text {min }}$ because the $k \mathrm{NN}$ classification algorithm is sensitive to the arbitrary choice of initial neighborhoods. To reproduce the same $k^{\mathrm{min}}$ use the set.seed() command in $\mathrm{R}$.
} 
that were analyzed in previous sections. Comparing Table 7 with Table 6 reveals that the $k \mathrm{NN}$ predictions are less accurate than the random forest predictions across all payment methods. However, a comparison with Table 4 (classification tree) and Table 5 (multinomial regression) shows improvements in prediction accuracy with respect to some (but not all) payment methods such as checks, BANP, OBBP, and account-to-account. These payment methods are used less frequently than cash, debit, and credit cards for which the $k \mathrm{NN}$ shows little or no improvement relative to the classification tree algorithm and the multinomial regression.

\section{Demonstration of the support vector machine algorithm}

Another commonly-used algorithm for analyzing supervised data is the support vector machine (SVM). The SVM algorithm is limited to a classification of two payment methods in the same way that a binomial regression is limited to a selection between only two options. Therefore, the SVM can be used to conduct research on cash users in order predict the use of cash versus non-cash payment methods. Similarly, the SVM can be used to research the use of card payments versus non-card payments. However, just like a binomial regression, there are no restrictions on the number of features (explanatory variables).

The SVM algorithm computes linear or nonlinear boundaries in the space of features. To illustrate the SVM, I simplify the regression model defined in (1) by splitting all the eight payment methods into two groups: cash payments versus non-cash payments. In addition, the number of features is restricted to "Amount" and "Age" because additional features cannot be drawn in two dimensions. Also, for the sake of illustration, the data are restricted to payment amounts not exceeding $\$ 40$ and to respondents not older than 80.

The black dots depicted in Figure 4 are 2,114 observed in-person cash payments. The dollar amount of each payment is given on the vertical axis. The age of the respondent who made the payment is given on the horizontal axis. Similarly, the red dots are 4,094 
observed in-person non-cash payments. ${ }^{10}$

The grey-shaded areas in Figure 4 are generated by the SVM algorithm. These areas are the combinations of the respondents' age and dollar amounts that are predicted by the SVM algorithm to be paid with cash. The non-shaded areas are the respondents' age and dollar amounts that are predicted to be paid with non-cash payment instruments.

The shaded areas in Figure 4 imply that the SVM algorithm predicts low-value inperson payments to be made with cash and high dollar amounts to be paid with non-cash methods. In addition, in-person cash payments are more likely to be used by consumers age 40 and older.

Finally, the SVM algorithm predicts payments in the amount near $\$ 10$ and $\$ 20$ to be paid with cash. These are illustrated by the shaded areas around the $\$ 10$ and $\$ 20$ dollar amounts drawn in Figure 4. Recall that the $\$ 10$ threshold was also predicted by the classification tree depicted in Figure 1. This happens because consumers tend to carry $\$ 10$ and $\$ 20$ bills in their wallets and also because most ATMs in the US dispense only \$20 currency notes. Note that there are also shaded areas around the $\$ 40$ payment amounts that can be paid with two $\$ 20$ bills. Footnote 5 provides a more detailed explanation for these observations.

\section{Conclusion}

Breiman (2001) discusses two goals for analyzing data: (i) prediction of what the responses to future input variables are going to be, and (ii) extracting information and intuition about how nature is associating the response variables with the input variables. As shown in earlier papers and in this article in the context of payment choice, some machine learning techniques excel in high prediction accuracy whereas logistic regressions excel in providing intuition on the nature of the connection between response and explanatory variables.

\footnotetext{
${ }^{10}$ The analysis in this section uses the $e 1071$ R-package and applies the svm function (with kernel = "radial" and cost $=0.49$ ). The somfit_tune function was used with kernel = "radial" over a sequence of potential cost parameters which yielded 0.49 as the optimal cost parameter.
} 
Statistical (machine) learning algorithms provide useful tools for understanding and predicting consumer payment choice. This article explores a few of these tools using the 2021 Survey and Diary of Consumer Payment Choice.

The advantages of using these tools relative to conventional regressions are:

(a) They can be implemented on datasets containing a large number of explanatory variables (called features in statistical learning).

(b) Do not rely on restrictive assumptions on the structure of the data for the sake of ensuring consistency of the estimated parameters.

(c) Provide useful visualization tools of the results, such as classification trees and variable importance plots.

(d) Some algorithms, such as random forest, provide more accurate out-of-sample predictions. In particular, they are extremely useful in predicting consumers' choice of payment methods that are not used very frequently (such as checks, prepaid cards, and account-to-account payments).

Some of the disadvantages of using machine learning algorithms include:

(i) Loss of interpretation power compared to the intuition that can be derived from coefficients and marginal effects estimated by linear and logistic regressions.

(ii) The tools are sensitive to their tuning parameters (regularization). However, it is important to bear in mind that regression results also have limitations because they are sensitive to the choice of model and control of confounders.

Anther commonly-used algorithm for analyzing supervised data, which is not demonstrated in this article, is neural networks. Neural network analysis attempts to mimic the way in which information transmission and decisions are made in the human brain. ${ }^{11}$ Running neural network algorithms generally requires heavier computational power.

To conclude, machine learning techniques and regression analyses could be viewed

\footnotetext{
${ }^{11}$ Liébana-Cabanillas and Lara-Rubio (2017) investigate which merchant characteristics affect merchants' decision to adopt a mobile payment system by comparing a logistic regression model with a neural network analysis. The authors show that the neural network algorithm is the most precise tool in this research for predicting the use of mobile payment systems in certain lines of business.
} 
as complements rather than as substitutes. Using tools from both disciplines could only enhance the reliability of algorithms that predict and interpret consumer payment choice.

\section{References}

Bajari, Patrick, Denis Nekipelov, Stephen Ryan, and Miaoyu Yang. 2015. "Machine Learning Methods for Demand Estimation." American Economic Review 105 (5):481-485.

Breiman, Leo. 2001. "Statistical Modeling: The Two Cultures." Statistical Science 16 (3):199-231.

Chen, Heng, Kim Huynh, and Oz Shy. 2019. "Cash Versus Card: Payment Discontinuities and the Burden of Holding Coins." Journal of Banking and Finance 99:192-201.

Cubides, Emily and Shaun O'Brien. 2022. “2022 Findings from the Diary of Consumer Payment Choice." Federal Reserve Bank of San Francisco.

Foster, Kevin, Claire Greene, and Joanna Stavins. Forthcoming. "2021 Survey and Diary of Consumer Payment Choice: Summary Results." Federal Reserve Bank of Atlanta, Research Data Report.

Henry, Christopher, Kim Huynh, and Angelika Welte. 2018. “2017 Methods-of-Payment Survey Report." Bank of Canada Staff Discussion Paper 2018-17.

Hillel, Tim, Michel Bierlaire, Mohammed Z.E.B. Elshafie, and Ying Jin. 2021. "A systematic review of machine learning classification methodologies for modelling passenger mode choice." Journal of Choice Modelling 38 (Article 100221).

James, Gareth, Daniela Witten, Trevor Hastie, and Robert Tibshirani. 2013. An Introduction to Statistical Learning, vol. 112. Springer.

Kleinberg, Jon, Jens Ludwig, Sendhil Mullainathan, and Ziad Obermeyer. 2015. "Prediction Policy Problems." American Economic Review 105 (5):491-495.

Liébana-Cabanillas, Francisco and Juan Lara-Rubio. 2017. "Predictive and Explanatory Modeling Regarding Adoption of Mobile Payment Systems." Technological Forecasting and Social Change 120:32-40.

Mullainathan, Sendhil and Jann Spiess. 2017. "Machine Learning: An Applied Econometric Approach." Journal of Economic Perspectives 31 (2):87-106. 
Shy, Oz. 2020. "How Currency Denomination and the ATM Affect the Way We Pay." Journal of Economics and Business 111 (Article 105908).

Varian, Hal. 2014. "Big data: New Tricks for Econometrics." Journal of Economic Perspectives 28 (2):3-27. 


\begin{tabular}{lrr} 
Variable & Weighted & Unweighted \\
\hline Number of respondents & 3137 & 3137 \\
\hline Age: $18-24(\%)$ & 6.32 & 3.41 \\
Age: $25-34(\%)$ & 23.21 & 13.17 \\
Age: $35-44(\%)$ & 17.39 & 20.72 \\
Age: 45-54 (\%) & 16.00 & 18.87 \\
Age: 55-64 (\%) & 16.30 & 20.27 \\
Age: 65 and older (\%) & 20.78 & 23.56 \\
\hline Gender: Female (\%) & 50.73 & 58.37 \\
\hline Education: Less than high school (\%) & 5.81 & 3.22 \\
Education: High school (\%) & 50.95 & 42.30 \\
Education: Associate or college (\%) & 25.92 & 33.54 \\
Education: Graduate (\%) & 17.32 & 20.94 \\
\hline Marital status: Married (\%) & 64.56 & 67.71 \\
\hline Household income: 0-30k (\%) & 16.03 & 16.58 \\
Household income: 30-60k (\%) & 20.46 & 19.99 \\
Household income: 60-90k (\%) & 20.72 & 22.25 \\
Household income: 90 and higher (\%) & 42.79 & 41.19 \\
\hline Household_size (Median) & 2.5 & 3.0
\end{tabular}

Table 1: Sample statistics by demographic groups.

Source: Author's computations from the 2021 Survey and Diary of Consumer Payment Choice. Notes: The table displays demographic information of 3,137 respondents. Weighted data are not used in the analysis and are brought here in order to demonstrate the similarities and differences between the sample variables and the actual US adult population. 


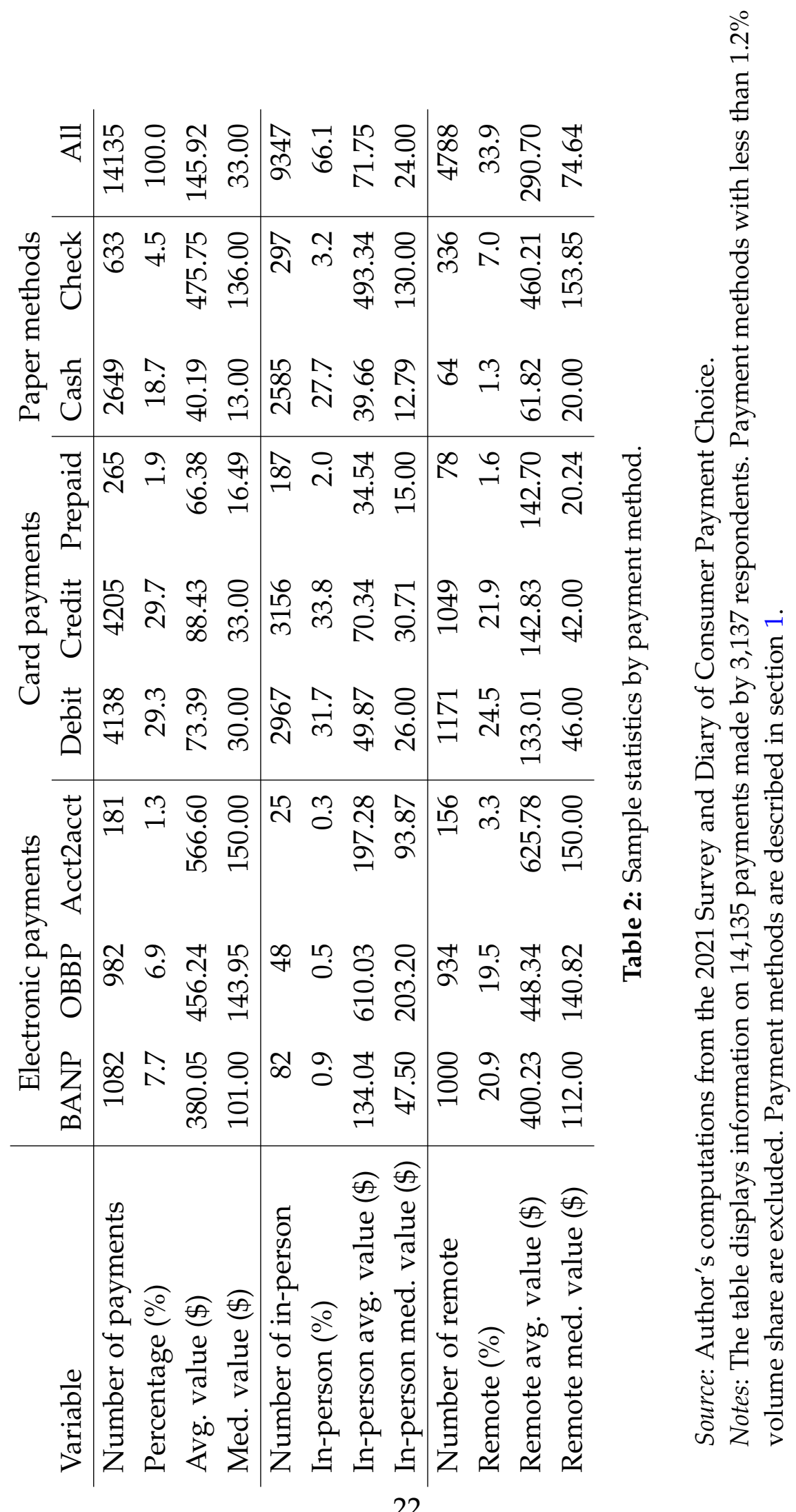




\begin{tabular}{|c|c|c|c|c|c|c|c|}
\hline & \multicolumn{7}{|c|}{ Dependent variable: } \\
\hline & $\begin{array}{c}\text { Check } \\
(1)\end{array}$ & $\begin{array}{c}\text { Debit } \\
(2)\end{array}$ & $\begin{array}{c}\text { Credit } \\
(3) \\
\end{array}$ & $\begin{array}{c}\text { Prepaid } \\
(4)\end{array}$ & $\begin{array}{c}\text { BANP } \\
(5) \\
\end{array}$ & $\begin{array}{c}\text { OBBP } \\
(6) \\
\end{array}$ & $\begin{array}{c}\text { Acct2acct } \\
(7)\end{array}$ \\
\hline Amount & $\begin{array}{c}0.004^{* * *} \\
(0.00004)\end{array}$ & $\begin{array}{l}0.002^{* * *} \\
(0.0001)\end{array}$ & $\begin{array}{l}0.003^{* * *} \\
(0.0001)\end{array}$ & $\begin{array}{c}0.001^{* * *} \\
(0.00000)\end{array}$ & $\begin{array}{c}0.004^{* * *} \\
(0.00004)\end{array}$ & $\begin{array}{l}0.004^{* * *} \\
(0.00004)\end{array}$ & $\begin{array}{l}0.004^{* * *} \\
(0.0001)\end{array}$ \\
\hline In_personYes & $\begin{array}{c}-3.873^{* * *} \\
(0.000)\end{array}$ & $\begin{array}{c}-2.670^{* * *} \\
(0.00000)\end{array}$ & $\begin{array}{c}-2.556^{* * *} \\
(0.00000)\end{array}$ & $\begin{array}{c}-2.695^{* * *} \\
(0.000)\end{array}$ & $\begin{array}{c}-6.149^{* * *} \\
(0.000)\end{array}$ & $\begin{array}{c}-6.536^{* * *} \\
(0.000)\end{array}$ & $\begin{array}{c}-5.252^{* * *} \\
(0.000)\end{array}$ \\
\hline Age & $\begin{array}{c}0.034^{* * *} \\
(0.00000)\end{array}$ & $\begin{array}{c}-0.028^{* * *} \\
(0.00001)\end{array}$ & $\begin{array}{c}-0.012^{* * *} \\
(0.00001)\end{array}$ & $\begin{array}{c}-0.019^{* * *} \\
(0.00000)\end{array}$ & $\begin{array}{c}-0.009^{* * *} \\
(0.00000)\end{array}$ & $\begin{array}{c}0.010^{* * *} \\
(0.00000)\end{array}$ & $\begin{array}{c}0.011^{* * *} \\
(0.00000)\end{array}$ \\
\hline GenderFemale & $\begin{array}{c}0.117^{* * *} \\
(0.000)\end{array}$ & $\begin{array}{c}0.404^{* * *} \\
(0.00000)\end{array}$ & $\begin{array}{c}-0.291^{* * *} \\
(0.00000)\end{array}$ & $\begin{array}{c}-0.022^{* * *} \\
(0.000)\end{array}$ & $\begin{array}{c}0.156^{* * *} \\
(0.00000)\end{array}$ & $\begin{array}{c}0.089^{* * *} \\
(0.00000)\end{array}$ & $\begin{array}{c}0.303^{* * *} \\
(0.000)\end{array}$ \\
\hline MaritalNot_married & $\begin{array}{c}-0.871^{* * *} \\
(0.000)\end{array}$ & $\begin{array}{c}-0.037^{* * *} \\
(0.00000)\end{array}$ & $\begin{array}{c}-0.504^{* * *} \\
(0.00000)\end{array}$ & $\begin{array}{c}0.542^{* * *} \\
(0.000)\end{array}$ & $\begin{array}{c}-0.0004^{* * *} \\
(0.00000)\end{array}$ & $\begin{array}{c}-0.495^{* * *} \\
(0.000)\end{array}$ & $\begin{array}{c}-0.422^{* * *} \\
(0.000)\end{array}$ \\
\hline EducHigh_school & $\begin{array}{c}0.833^{* * *} \\
(0.000)\end{array}$ & $\begin{array}{c}0.425^{* * *} \\
(0.00000)\end{array}$ & $\begin{array}{c}1.816^{* * *} \\
(0.00000)\end{array}$ & $\begin{array}{c}0.584^{* * *} \\
(0.000)\end{array}$ & $\begin{array}{l}1.117^{* * *} \\
(0.00000)\end{array}$ & $\begin{array}{l}1.589^{* * *} \\
(0.00000)\end{array}$ & $\begin{array}{c}2.199^{* * *} \\
(0.000)\end{array}$ \\
\hline EducAssoc_or_college & $\begin{array}{c}0.982^{* * *} \\
(0.000)\end{array}$ & $\begin{array}{c}0.380^{* * *} \\
(0.00000)\end{array}$ & $\begin{array}{l}2.745^{* * *} \\
(0.00000)\end{array}$ & $\begin{array}{c}1.006^{* * *} \\
(0.000)\end{array}$ & $\begin{array}{c}1.652^{* * *} \\
(0.00000)\end{array}$ & $\begin{array}{c}2.155^{* * *} \\
(0.000)\end{array}$ & $\begin{array}{c}2.734^{* * *} \\
(0.000)\end{array}$ \\
\hline EducMA+ & $\begin{array}{c}0.849^{* * *} \\
(0.000)\end{array}$ & $\begin{array}{c}0.167^{* * *} \\
(0.00000)\end{array}$ & $\begin{array}{l}2.988^{* * *} \\
(0.00000)\end{array}$ & $\begin{array}{c}0.866^{* * *} \\
(0.000)\end{array}$ & $\begin{array}{l}1.599^{* * *} \\
(0.00000)\end{array}$ & $\begin{array}{c}1.922^{* * *} \\
(0.000)\end{array}$ & $\begin{array}{c}2.299^{* * *} \\
(0.000)\end{array}$ \\
\hline HH_income & $\begin{array}{c}0.00000^{* * *} \\
(0.00000)\end{array}$ & $\begin{array}{c}0.00000^{* * *} \\
(0.00000)\end{array}$ & $\begin{array}{c}0.00000^{* * *} \\
(0.00000)\end{array}$ & $\begin{array}{c}0.00000^{* * *} \\
(0.00000)\end{array}$ & $\begin{array}{c}0.00000^{* * *} \\
(0.00000)\end{array}$ & $\begin{array}{c}0.00000^{* * *} \\
(0.00000)\end{array}$ & $\begin{array}{c}0.00000^{* * *} \\
(0.00000)\end{array}$ \\
\hline HH_size & $\begin{array}{c}-0.066^{* * *} \\
(0.00000)\end{array}$ & $\begin{array}{c}-0.018^{* * *} \\
(0.00000)\end{array}$ & $\begin{array}{c}-0.101^{* * *} \\
(0.00000)\end{array}$ & $\begin{array}{c}0.075^{* * *} \\
(0.00000)\end{array}$ & $\begin{array}{c}-0.032^{* * *} \\
(0.00000)\end{array}$ & $\begin{array}{c}-0.100^{* * *} \\
(0.00000)\end{array}$ & $\begin{array}{c}-0.108^{* * *} \\
(0.00000)\end{array}$ \\
\hline Constant & $\begin{array}{c}-1.304^{* * *} \\
(0.00000)\end{array}$ & $\begin{array}{l}3.382^{* * *} \\
(0.00000)\end{array}$ & $\begin{array}{c}1.222^{* * *} \\
(0.00000)\end{array}$ & $\begin{array}{c}-0.454^{* * *} \\
(0.000)\end{array}$ & $\begin{array}{c}1.163^{* * *} \\
(0.00000)\end{array}$ & $\begin{array}{c}0.173^{* * *} \\
(0.00000)\end{array}$ & $\begin{array}{c}-2.503^{* * *} \\
(0.000)\end{array}$ \\
\hline Akaike Inf. Crit. & $31,124.490$ & $31,124.490$ & $31,124.490$ & $31,124.490$ & $31,124.490$ & $31,124.490$ & $31,124.490$ \\
\hline
\end{tabular}

Table 3: Multinomial regression: Estimated coefficients.

Source: Author's computations from the 2021 Survey and Diary of Consumer Payment Choice. Notes: Cash is the reference dependent variable. To maintain consistency throughout this article, HH_income is measured in dollar units which explains why the coefficients are so tiny. The regression is based on 11,308 payment observations in training subsample. 


\begin{tabular}{rrrrrrrrr} 
Payment method & Cash & Check & Debit & Credit & Prepaid & BANP & OBBP & Acct2acct \\
\hline Cash & 258 & 8 & 96 & 72 & 13 & 5 & 0 & 1 \\
Check & 0 & 0 & 0 & 0 & 0 & 0 & 0 & 0 \\
Debit & 135 & 50 & 456 & 210 & 31 & 78 & 68 & 11 \\
Credit & 142 & 44 & 246 & 484 & 13 & 27 & 29 & 6 \\
Prepaid & 0 & 0 & 0 & 0 & 0 & 0 & 0 & 0 \\
BANP & 0 & 5 & 24 & 23 & 1 & 32 & 24 & 7 \\
OBBP & 1 & 33 & 16 & 48 & 0 & 54 & 67 & 9 \\
Acct2acct & 0 & 0 & 0 & 0 & 0 & 0 & 0 & 0 \\
\hline Total actual & 536 & 140 & 838 & 837 & 58 & 196 & 188 & 34 \\
Correct predictions & 258 & 0 & 456 & 484 & 0 & 32 & 67 & 0 \\
Correct rate (\%) & 48 & 0 & 54 & 58 & 0 & 16 & 36 & 0
\end{tabular}

Table 4: Confusion matrix for the classification tree.

Source: Author's computations from the 2021 Survey and Diary of Consumer Payment Choice. Notes: Based on 11,308 training payment observations and 2,827 testing payment observations. The main diagonal displays number of correct predictions.

\begin{tabular}{rrrrrrrrr} 
Payment method & Cash & Check & Debit & Credit & Prepaid & BANP & OBBP & Acct2acct \\
\hline Cash & 160 & 14 & 107 & 88 & 9 & 0 & 2 & 1 \\
Check & 1 & 9 & 3 & 3 & 0 & 0 & 2 & 0 \\
Debit & 173 & 35 & 412 & 183 & 31 & 73 & 57 & 11 \\
Credit & 199 & 42 & 290 & 517 & 17 & 52 & 56 & 9 \\
Prepaid & 0 & 0 & 0 & 0 & 0 & 0 & 0 & 0 \\
BANP & 0 & 9 & 13 & 14 & 0 & 33 & 27 & 7 \\
OBBP & 3 & 31 & 13 & 32 & 1 & 38 & 44 & 6 \\
Acct2acct & 0 & 0 & 0 & 0 & 0 & 0 & 0 & 0 \\
\hline Total actual & 536 & 140 & 838 & 837 & 58 & 196 & 188 & 34 \\
Correct predictions & 160 & 9 & 412 & 517 & 0 & 33 & 44 & 0 \\
Correct rate (\%) & 30 & 6 & 49 & 62 & 0 & 17 & 23 & 0
\end{tabular}

Table 5: Confusion matrix for the multinomial regression.

Source: Author's computations from the 2021 Survey and Diary of Consumer Payment Choice. Notes: Based on 11,308 training payment observations and 2,827 testing payment observations. The main diagonal displays number of correct predictions. 


\begin{tabular}{rrrrrrrrr} 
Payment method & Cash & Check & Debit & Credit & Prepaid & BANP & OBBP & Acct2acct \\
\hline Cash & 287 & 8 & 91 & 73 & 14 & 4 & 2 & 0 \\
Check & 0 & 46 & 6 & 7 & 2 & 5 & 8 & 1 \\
Debit & 121 & 26 & 568 & 114 & 17 & 36 & 32 & 5 \\
Credit & 125 & 39 & 126 & 582 & 16 & 33 & 22 & 1 \\
Prepaid & 1 & 0 & 2 & 3 & 6 & 0 & 0 & 0 \\
BANP & 1 & 12 & 24 & 27 & 1 & 87 & 27 & 10 \\
OBBP & 1 & 9 & 19 & 31 & 2 & 29 & 95 & 10 \\
Acct2acct & 0 & 0 & 2 & 0 & 0 & 2 & 2 & 7 \\
\hline Total actual & 536 & 140 & 838 & 837 & 58 & 196 & 188 & 34 \\
Correct predictions & 287 & 46 & 568 & 582 & 6 & 87 & 95 & 7 \\
Correct rate (\%) & 54 & 33 & 68 & 70 & 10 & 44 & 51 & 21
\end{tabular}

Table 6: Confusion matrix for the random forest.

Source: Author's computations from the 2021 Survey and Diary of Consumer Payment Choice. Notes: Based on 11,308 training payment observations and 2,827 testing payment observations. The main diagonal displays number of correct predictions.

\begin{tabular}{rrrrrrrrr} 
Payment method & Cash & Check & Debit & Credit & Prepaid & BANP & OBBP & Acct2acct \\
\hline Cash & 166 & 22 & 137 & 95 & 9 & 7 & 3 & 1 \\
Check & 1 & 14 & 9 & 13 & 1 & 8 & 7 & 1 \\
Debit & 150 & 30 & 369 & 158 & 28 & 47 & 41 & 9 \\
Credit & 213 & 52 & 261 & 491 & 15 & 43 & 34 & 10 \\
Prepaid & 0 & 0 & 0 & 0 & 0 & 0 & 0 & 0 \\
BANP & 4 & 8 & 26 & 36 & 2 & 47 & 30 & 6 \\
OBBP & 2 & 13 & 34 & 43 & 3 & 43 & 69 & 6 \\
Acct2acct & 0 & 1 & 2 & 1 & 0 & 1 & 4 & 1 \\
\hline Total actual & 536 & 140 & 838 & 837 & 58 & 196 & 188 & 34 \\
Correct predictions & 166 & 14 & 369 & 491 & 0 & 47 & 69 & 1 \\
Correct rate (\%) & 31 & 10 & 44 & 59 & 0 & 24 & 37 & 3
\end{tabular}

Table 7: Confusion matrix for the $k \mathrm{NN}$.

Source: Author's computations from the 2021 Survey and Diary of Consumer Payment Choice. Notes: Based on 11,308 training payment observations and 2,827 testing payment observations. The main diagonal displays number of correct predictions. 


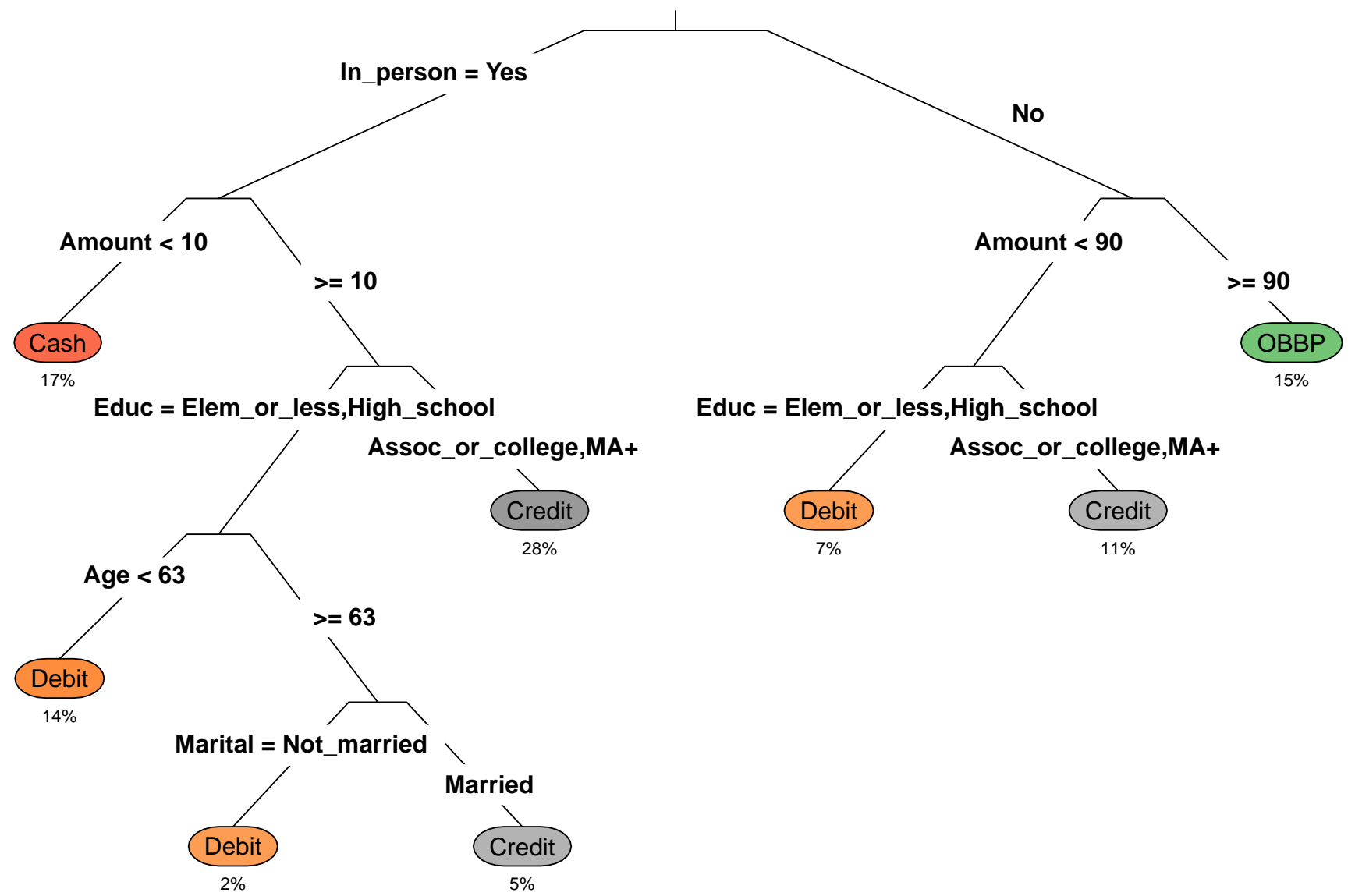

Figure 1: Classification tree: Predicting the use of payment methods based on whether the payment is made in-person, payment dollar amount, and some other consumer demographic variables.

Source: Author's computations from the 2021 Survey and Diary of Consumer Payment Choice. Notes: Based on 14,135 payment observations made by 3,137 respondents. For illustrative purposes, the displayed tree has been pruned to have only 7 splits instead of 23 splits that locally minimize the average cross-validation error. 


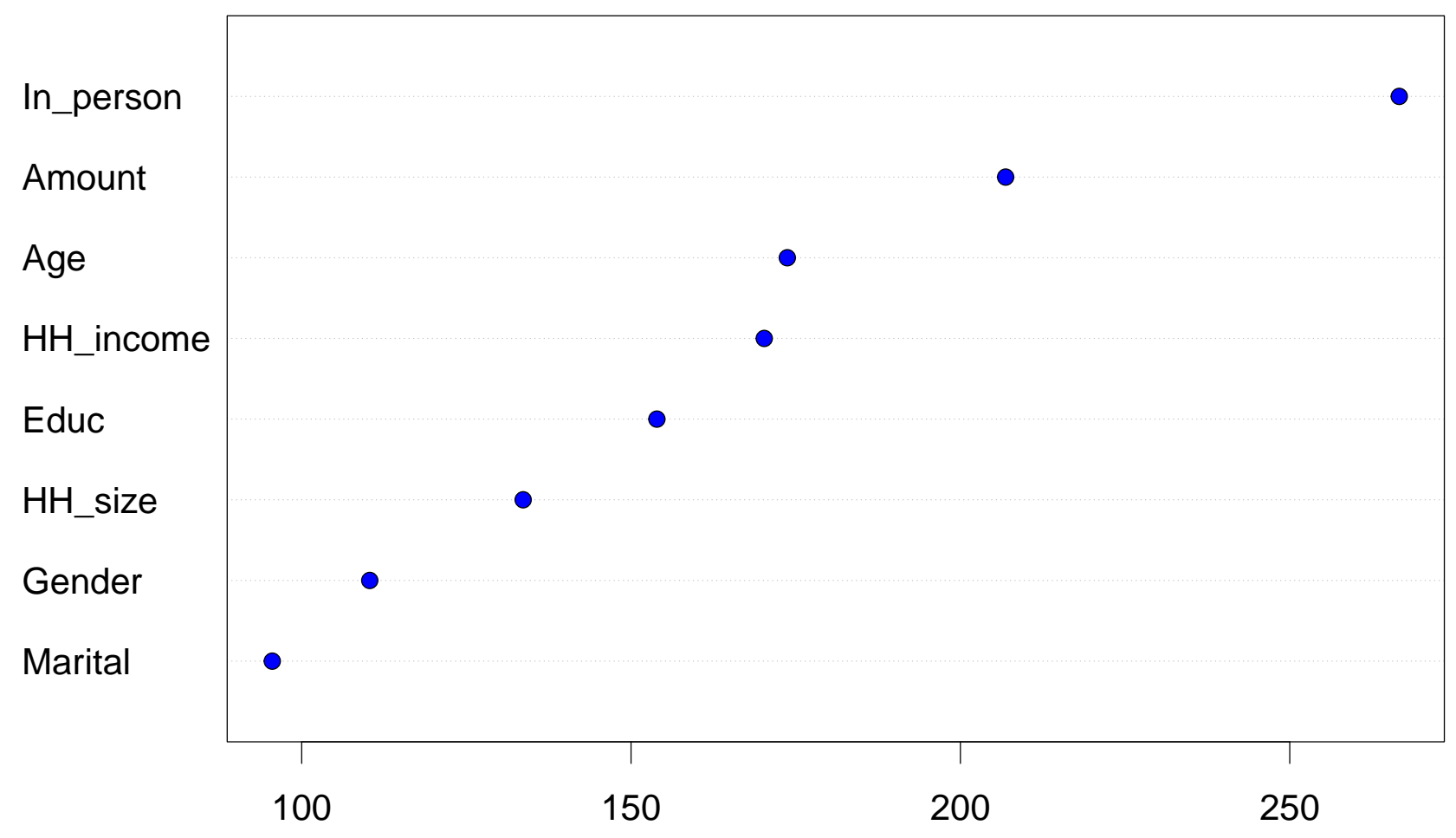

Figure 2: Variable importance plot (VIP) generated by random forest.

Source: Author's computations from the 2021 Survey and Diary of Consumer Payment Choice. Notes: Based on 14,135 payment observations. 

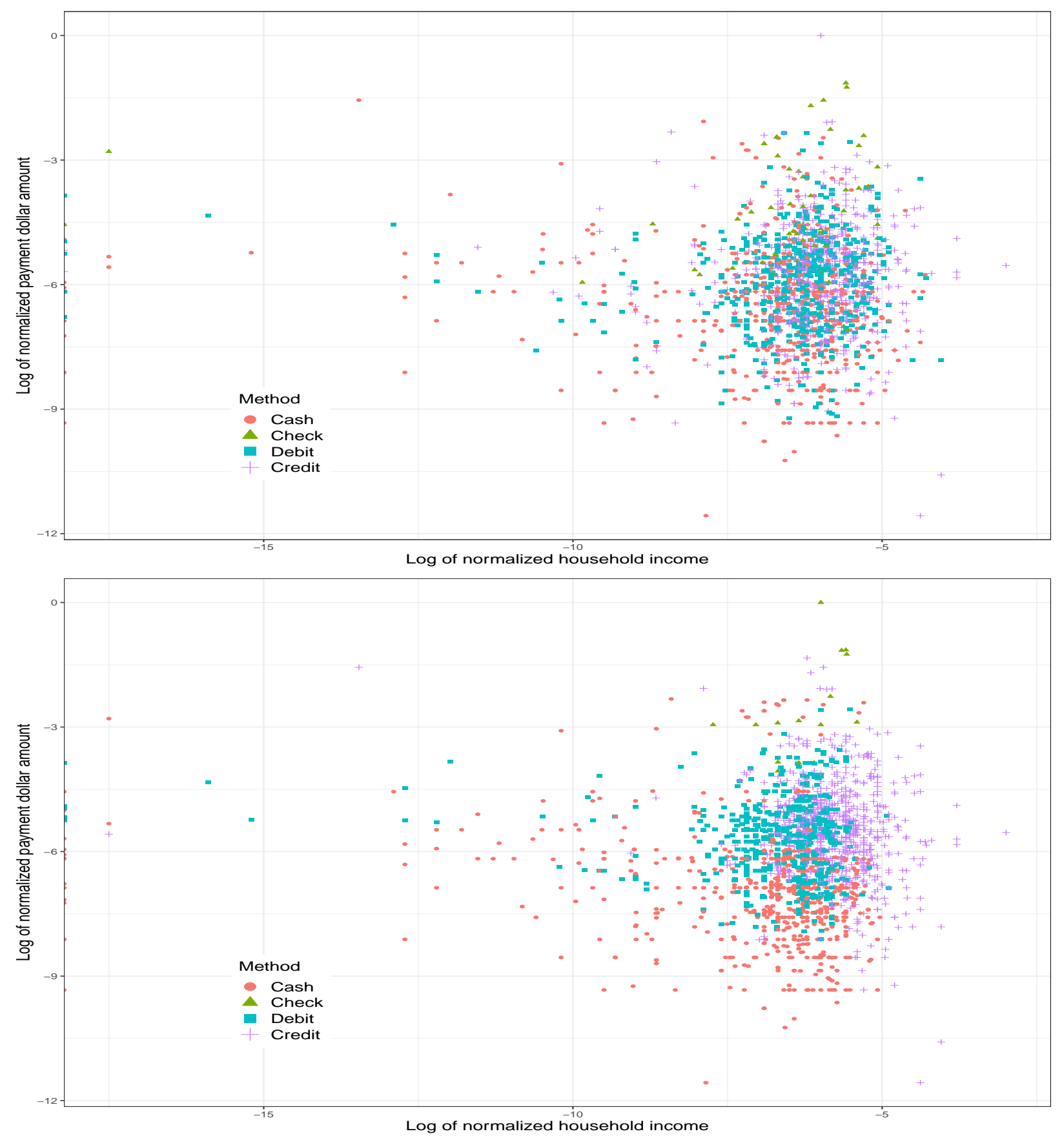

Figure 3: $k \mathrm{NN}$ classifications of in-person payment methods in the space of two features: Amount and household income.

Source: Author's computations from the 2021 Survey and Diary of Consumer Payment Choice. Notes: Based on 7,477 training in-person payment observations and 1,870 testing in-person payment observations. Top: Actual in-person payment observations in the testing data. Bottom: Predicted payment methods based on two features in the testing data. 


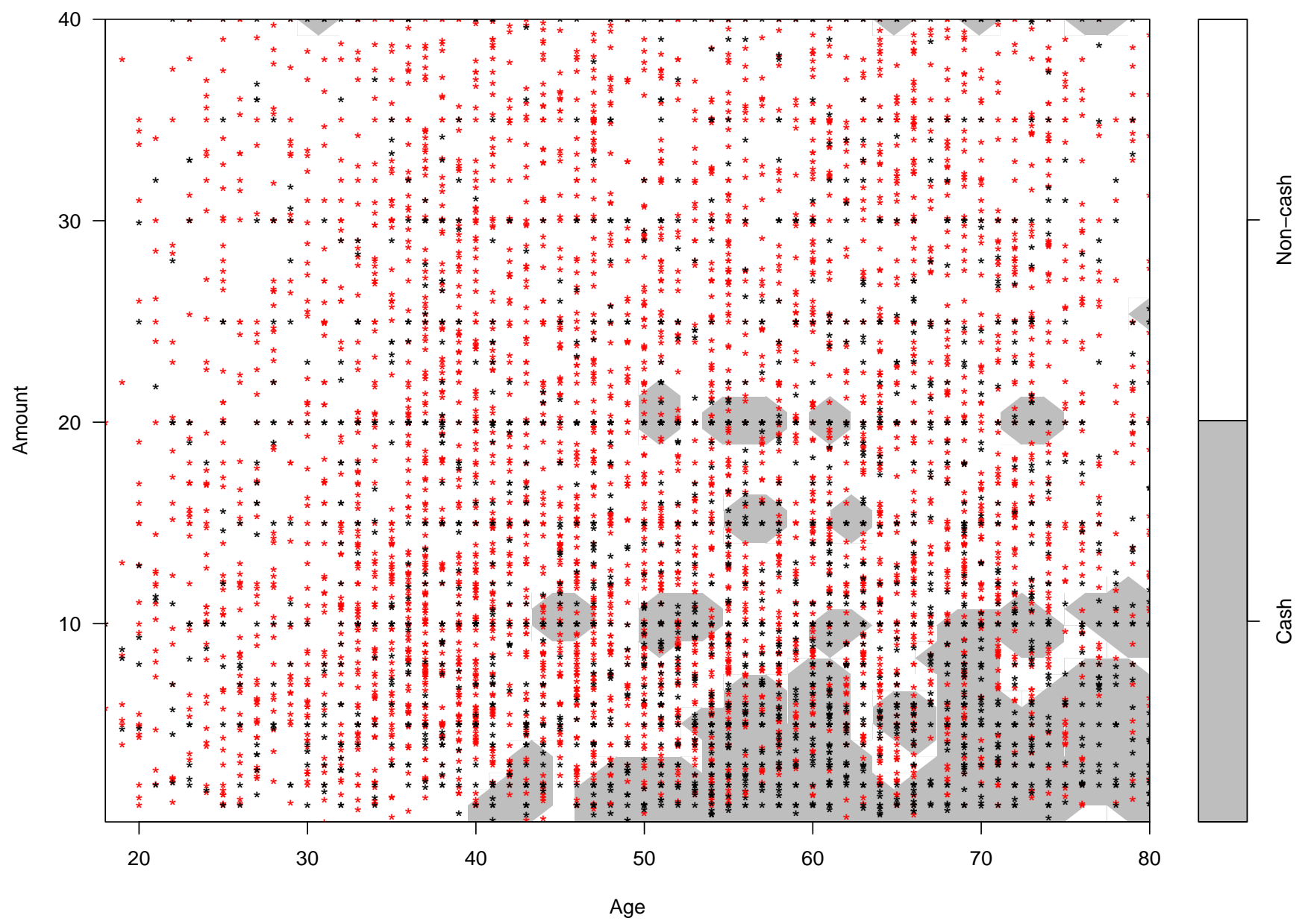

Figure 4: SVM payment method classifications in the space of two features: Amount and age.

Source: Author's computations from the 2021 Survey and Diary of Consumer Payment Choice. Notes: Based on 6,208 (2,114 cash and 4,094 non-cash) in-person payment observations with amounts not exceeding $\$ 40$ by respondents not older than 80 . 